\title{
Verbal memory improvement in first-episode psychosis APOE- $\varepsilon 4$ carriers: a pleiotropic effect?
}

This article was published in the following Dove Press journal:

Neuropsychiatric Disease and Treatment

\author{
Fidel Vila-Rodriguez' \\ Donna J Lang ${ }^{2}$ \\ Heather Baitz ${ }^{3}$ \\ Kristina Gicas ${ }^{3}$ \\ Allen E Thorton ${ }^{3}$ \\ Thomas S Ehmann' \\ Geoff N Smith' \\ Alasdair M Barr ${ }^{4}$ \\ Ivan J Torres' \\ Lili C Kopala' \\ G William MacEwan' \\ Daniel J Müller ${ }^{5}$ \\ James L Kennedy ${ }^{5}$ \\ William G Honer \\ 'Department of Psychiatry, ${ }^{2}$ Division \\ of Neuroradiology, Department of \\ Radiology, The University of British \\ Columbia, Vancouver, ${ }^{3}$ Department \\ of Psychology, Simon Fraser \\ University, Burnaby, ${ }^{4}$ Department of \\ Anesthesiology, Pharmacology and \\ Therapeutics, The University of \\ British Columbia, Vancouver, BC, \\ ${ }^{5}$ Department of Psychiatry, Centre \\ for Mental Health and Addictions, \\ University of Toronto, Toronto, \\ ON, Canada
}

Background: Verbal memory impairment is a core feature in schizophrenia even at early stages of the disease, but its etiopathogenesis is not fully understood. The APOE- $\varepsilon 4$ is the main genetic risk factor for late-onset Alzheimer's disease. Our primary goal was to ascertain whether $A P O E-\varepsilon 4$ status had a pleiotropic effect in early stages of the illness.

Participants and methods: A total of 86 first-episode psychosis (FEP) outpatients and 39 healthy volunteers were recruited. Demographic and clinical data, APOE genotyping, and a neuropsychological test battery including the California Verbal Learning Test - second edition (CVLT-II) were administered and assessed at study entry and at 1-year follow-up. Data were analyzed using mixed-model repeated measures, where the dependent variable was verbal memory indexed by California Verbal Learning Test (CVLT) Trials 1-5 total recall score.

Results: FEP- $A P O E-\varepsilon 4$ carriers and FEP- $A P O E-\varepsilon 4$ noncarriers had similar symptom severity, clinical outcomes, premorbid and current intelligence quotient, and exposure to antipsychotics. There was a main effect of group on CVLT $1-5$ (FEP $=43.30$ vs control $=58.25 ; F[1$, 119.7] $=42.97 ; P<0.001)$ as well as an $A P O E-\varepsilon 4$ by group by time $(F[4,116.2]=2.73, P=0.033)$ interaction with only FEP-APOE- $\varepsilon 4$ carriers showing improved verbal memory at follow-up. Conclusion: Our study is the first to report improvement in verbal memory in persons afflicted by FEP who are $A P O E-\varepsilon 4$ carriers and replicates the prominent verbal memory deficits present in FEP. Our work provides further evidence pointing to an antagonistic pleiotropic effect of $A P O E-\varepsilon 4$ in neuropsychiatric disorders. Our results merit further research into antagonistic pleiotropic effects in schizophrenia.

Keywords: APOE, verbal memory, antagonistic pleiotropy, first-episode psychosis, schizophrenia

\section{Introduction}

In spite of not been included as a diagnostic criterion in current diagnostic tools, cognitive impairment is a core feature in schizophrenia and first-episode psychosis (FEP) and has been closely linked to functional disability. ${ }^{1,2}$ A pivotal meta-analysis investigating cognitive deficits in first-episode schizophrenia showed moderate-tosevere cognitive deficits across all neuropsychological domains tested with effect sizes ranging from -0.64 to $-1.20 .{ }^{3}$ Deficits in verbal memory were among the most severely affected domains, which parallels results found in chronic populations. ${ }^{4}$ Despite vast, consistent, and robust evidence of significant verbal memory deficits in schizophrenia and FEP, the etiopathogenesis of these deficits has not yet been fully elucidated.

One potential polymorphism of interest in schizophrenia-associated verbal memory impairment is the $A P O E$ gene. The $A P O E$ gene is present in the following three main alleles in humans: $A P O E$ varepsilon 3, 79\%; $A P O E$ varepsilon 4 ( $A P O E-\varepsilon 4), 14 \%$; and $A P O E$ varepsilon $2,8 \% .^{5} A P O E$ codifies for a protein, APOE, that has a critical role
Correspondence: Fidel Vila-Rodriguez Department of Psychiatry, University of British Columbia, Non-Invasive Neurostimulation Laboratory \& Schizophrenia Program at UBC Hospital, Detwiller Pavilion, 2255 Wesbrook Mall, Vancouver, BC V6T 2AI, Canada

Tel + I 6048270175

Email fildelvil@mail.ubc.ca 
in synaptogenesis, neurite outgrowth, and membrane repair and maintenance. ${ }^{6-9}$ In addition, $A P O E-\varepsilon 4$ is the main genetic risk factor for late-onset Alzheimer's disease where cognitive impairment is the core manifestation of the disorder ${ }^{10}$ and has been associated with verbal memory impairment in this condition. ${ }^{11,12}$ Furthermore, APOE- $\varepsilon 4$ carrier status has been associated with the severity of neuropathological findings such as hippocampal neurofibrillary tangles, neurite plaque density, and severity of dementia in brains from schizophrenia patients, ${ }^{13}$ and some studies report earlier onset of schizophrenia ${ }^{14}$ and association to positive symptoms ${ }^{15}$ or to negative symptoms. ${ }^{16}$ Of note, there are no studies reporting on the effect of $A P O E-\varepsilon 4$ on verbal memory in psychosis.

The relationship between $A P O E-\varepsilon 4$, schizophrenia, and verbal memory is likely to be complex. First, although early studies and meta-analysis showed increased APOE- $\varepsilon 4$ carriers status associated with schizophrenia, ${ }^{15,17}$ this finding was not universally replicated ${ }^{18-20}$ and the most recent metaanalysis by González-Castro et $\mathrm{al}^{20}$ did not replicate earlier meta-analysis results. Second, recent research investigating the course of cognitive function and genetic risk in psychiatric disorders showed that genetic-associated cognitive impairment was worse in schizophrenia only in older age, but not in young populations, pointing to a pleiotropic effect. ${ }^{21}$ In this regard, divergent results depending on the life stage could point to an antagonistic pleiotropic effect. ${ }^{22}$ For an antagonistic pleiotropic effect to occur, a gene has to show that it impacts fitness differently during different life stages. There is prior literature showing that APOE- $\varepsilon 4$ is associated with increased fertility as well as better cognition in young healthy adults, ${ }^{23-26}$ and there is ample evidence that APOE- $\varepsilon 4$ plays a detrimental role on cognition in Alzheimer's disease, leading to hypothesize that APOE- $\varepsilon 4$ has such an antagonistic pleiotropic effect. ${ }^{27}$

To further our understanding of the potential role of $A P O E-\varepsilon 4$ in the etiopathogenesis of verbal memory impairment in FEP, we conducted a prospective cohort study in recent onset psychosis disorders. Our primary goal was to ascertain whether $A P O E-\varepsilon 4$ status had a pleiotropic effect in early stages of the disorder as prior research has shown an antagonistic pleiotropic effect of $A P O E-\varepsilon 4$ in young healthy volunteers.

\section{Participants and methods Methods}

The research protocol was approved by the University of British Columbia and Vancouver Coastal Health Authority Clinical Research Ethics Boards and was in accordance with Tri-Council Policy. Written informed consent was obtained from all participants after the procedures had been fully explained.

Participants were recruited from a longitudinal study from an Early Psychosis Intervention Program serving a catchment area population of 640,000 in the Vancouver area. All patients referred to the program were approached to enter the study. Participants had $<8$ weeks of total lifetime exposure to antipsychotic treatment at the time of cognitive assessment (refer further details in Supplementary material).

\section{Participants}

The sample comprised 86 FEP subjects and 39 healthy volunteers (control [CTRL]). The FEP group consisted of schizophrenia patients $(n=44)$, schizoaffective patients $(n=13)$, affective psychosis patients $(n=17)$, and other psychosis patients $(n=12)$. FEP and CTRL groups were similar in mean age, sex, ethnicity distribution, and prevalence of APOE- $\varepsilon 4$ carriers (Table 1).

A summary of demographic and clinical characteristics comparing the FEP $A P O E-\varepsilon 4$ carriers group (FEP-APOE- $\varepsilon 4+$ ) vs the FEP non- $A P O E-\varepsilon 4$ carrier group (FEP- $A P O E-\varepsilon 4-)$ at baseline is presented in Table 2 . In summary, both groups were similar in mean age, mean years of education, and sex

Table I Demographic characteristics and premorbid and current IQ of the sample

\begin{tabular}{|c|c|c|c|}
\hline Characteristics & FEP $(n=86)$ & CTRL $(n=39)$ & \\
\hline Male:female (\% male) & $59: 27(68.6 \%)$ & $25: 14(64.1 \%)$ & NS $\left(\chi^{2}=0.25 ; \mathrm{df}=\mathrm{I} ; P=0.62\right)$ \\
\hline Age (years), mean (SD) & $20.4 I(4.2)$ & $20.8(3.7)$ & NS $(t=-0.53 ; \mathrm{df}=123 ; P=0.60)$ \\
\hline Ethnicity, Cau:Asi:others (\% Cau) & $60: 13: 13(69.8 \%)$ & $26: 7: 6(66.7 \%)$ & $\operatorname{NS}\left(\chi^{2}=0.17 ; \mathrm{df}=2 ; P=0.92\right)$ \\
\hline APOE- $\varepsilon 4$ carriers, ${ }^{\mathrm{a}} \mathrm{n}(\%)$ & $29(33.7 \%)$ & $8(20.5 \%)$ & $\operatorname{NS}\left(\chi^{2}=2.25 ; \mathrm{df}=\mathrm{I} ; P=0.13\right)$ \\
\hline Education (years), mean (SD) & $11.56(1.7)$ & $13.38(2.05)$ & $(t=-5.2 ; \mathrm{df}=\mid 23 ; P<0.00 \mathrm{I})$ \\
\hline NAART FSIQ, mean (SD) & $100.45(8.01)$ & $107.54(6.75)$ & $(t=-4.80 ; \mathrm{df}=123 ; P<0.00 \mathrm{I})$ \\
\hline K-BIT IQ, mean (SD) & $94.67(11.7)$ & $108.5(9.9)$ & $(t=-6.35 ; \mathrm{df}=\mathrm{I} 2 \mathrm{I} ; P<0.00 \mathrm{I})$ \\
\hline
\end{tabular}

Note: ${ }^{a}$ One APOE- $\varepsilon 2 / 4$ was excluded.

Abbreviations: Asi, Asian; Cau, Caucasian; CTRL, control; FEP, first-episode psychosis; FSIQ, Full Scale IQ; K-BIT, Kauffman Brief Intelligence Test; IQ, intelligence quotient; NAART, North American Reading Test. 
Table 2 Clinical characteristics of first-episode psychosis patients, non-APOE- $\varepsilon 4$ carriers vs APOE- $\varepsilon 4$ carriers

\begin{tabular}{|c|c|c|c|}
\hline Characteristics & $\begin{array}{l}\text { Non-APOE- } \varepsilon 4 \\
(n=57)\end{array}$ & $\begin{array}{l}\text { APOE- } \varepsilon 4 \text { carriers } \\
(\mathrm{n}=29)\end{array}$ & Statistics \\
\hline Male:female (\% male) & $40: 17(70.2 \%)$ & $19: 10(65 \%)$ & $\operatorname{NS}\left(\chi^{2}=0.19 ; d f=I ; P=0.66\right)$ \\
\hline Age (years), mean (SD) & $20.6(4.4)$ & $20.07(3.8)$ & NS $(t=-0.53 ; d f=84 ; P=0.6)$ \\
\hline Ethnicity, Cau:Asi:others (\% Cau) & $39: 9: 9(68.4 \%)$ & $21: 4: 4(72.4 \%)$ & $\operatorname{NS}\left(\chi^{2}=0.145 ; d f=2 ; P=0.93\right)$ \\
\hline Education (years), mean (SD) & II.5 (I.6) & $11.6(1.9)$ & NS $(t=0.24 ; d f=84 ; P=0.8)$ \\
\hline NAART FSIQ, mean (SD) & $101.0(7.8)$ & $99.4(8.5)$ & NS $(t=-0.86 ; d f=84 ; P=0.4)$ \\
\hline K-BIT IQ, mean (SD) & $95.6(10.7)$ & $92.8(13.4)$ & NS $(t=-1.01 ; d f=83 ; P=0.3)$ \\
\hline Diagnoses, SCZ:SCZ_A:AfP:others (\% SCZ) & 29:9:1।:8 (51\%) & $15: 4: 6: 4(52 \%)$ & NS $\left(\chi^{2}=2.8 ; d f=4 ; P=0.59\right)$ \\
\hline \multicolumn{4}{|l|}{ PANSS scores, mean (SD) } \\
\hline Positive & $19.3(6.5)$ & $19.1(5.1)$ & NS $(t=0.36 ; d f=83 ; P=0.7)$ \\
\hline Negative & I8.I (6.9) & $19.1(5.1)$ & \\
\hline General & $4 I . I(9.3)$ & $41.8(7.3)$ & \\
\hline Total & $78.5(19.5)$ & $80.03(15.8)$ & \\
\hline CGI, mean (SD) & $4.16(0.9)$ & $4.36(0.7)$ & NS $(t=0.39 ; d f=82 ; P=0.3)$ \\
\hline Age first decline, mean (SD) & $17.7(4.6)$ & $17.6(4.5)$ & NS $(t=0.82 ; d f=87 ; P=0.9)$ \\
\hline \multicolumn{4}{|l|}{ Onset of symptoms } \\
\hline Age first psychosis, mean (SD) & $19.0(4.7)$ & $19.0(4.7)$ & NS $(t=0.48 ; d f=78 ; P=0.9)$ \\
\hline Duration of untreated psychosis (months), mean (SD) & $22.3(27.2)$ & $17.2(22.2)$ & NS $(t=0.36 ; d f=76 ; P=0.4)$ \\
\hline \multicolumn{4}{|l|}{ Medications } \\
\hline Risperidone/olanzapine/others/missing & $17 / 16 / 3 / 8$ & $|4 / 6 / 0 /|$ & NS $\left(\chi^{2}=3.6 ; d f=2 ; P=0.17\right)$ \\
\hline Chlorpromazine equivalent dose, mean (SD) & $210.6(196.5)$ & I 40.7 ( 107.9$)$ & NS $(t=1.67 ; d f=69 ; P=0.1)$ \\
\hline AP naïve, $\mathrm{n}(\%)$ & $13(26 \%)$ & $8(29 \%)$ & NS (Fisher; $P=0.52$ ) \\
\hline
\end{tabular}

Abbreviations: AfP, affective psychosis; AP, antipsychotic; Asi, Asian; Cau, Caucasian; CGI, clinical global impression; GAF, Global Assessment of Functioning; FSIQ, Full Scale IQ; IQ, intelligence quotient; K-BIT, Kauffman Brief Intelligence Test; NAART, North American Reading Test; NS, non-significant; PANSS, Positive and Negative Syndrome Scale; SCZ, schizophrenia; SCZ_A, schizoaffective; SOFAS, Social and Occupational Functioning Assessment Scale.

distribution. In addition, both groups had similar premorbid and current estimated intelligence quotient (IQ). The two groups were similar with respect to symptom severity, level of functioning, and medication status.

\section{APOE genotyping}

Genotyping of the APOE rs7412 and rs429358 polymorphisms was performed using standard ABI (Thermo Fisher Scientific, Waltham, MA, USA) single nucleotide polymorphism genotyping assays. Genotype calls were performed manually. Laboratory personnel blind to demographic and phenotypic information independently verified the results.

To ensure quality CTRL, $10 \%$ of the samples were randomly re-genotyped with a $100 \%$ concordance rate.

\section{Neuropsychological assessment}

Neuropsychological testing was completed by trained research assistants under the supervision of a clinical neuropsychologist (TSE). Cognition was evaluated at the baseline and 9-12-month time points. Premorbid intellectual functioning was estimated at baseline with the North American Adult Reading Test. ${ }^{28}$ Current IQ was estimated at baseline with the Kaufman Brief Intelligence Test. ${ }^{29}$ Verbal memory was assessed using the California Verbal Learning Test - second edition (CVLT-II); ${ }^{30}$ we used two alternate versions of the CVLT-II to minimize practice effects, ${ }^{31}$ and participants were randomly assigned to receive those versions in a counterbalanced way.

\section{Statistical analyses}

Statistical analyses were performed with SPSS (Version 24.0; IBM Corporation, Armonk, NY, USA). Data exploratory analysis was used to ascertain the characteristics of the distribution of the variables, including testing for normality.

Profile analysis, a particular application of repeated measures multiple analysis of covariance, is a multivariate technique for analyzing a set of measures across and between groups. ${ }^{32}$ Six primary CVLT-II ${ }^{30}$ standard scores were treated as within-subjects variables analogous to repeated measures (ie, standardized CVLT-II scores on Trial 1, Trial 5, short delay free recall, short delay cued recall, long delay free recall, and long delay cued recall), and groups (FEP and CTRL) were the between-subjects variables. Profile analysis tested for parallelism, separation, and flatness of the plotted patient and CTRL CVLT-II score profiles. $^{32}$ Analysis of variance was used to test the null hypothesis of equal means of verbal memory across four types of medication (dependent variable CVLT-II Trials 
$1-5$, independent variable type of medication at baseline). CVLT-II standardized scores were used.

We used SPSS mixed procedure to perform a mixed-model repeated measures (MMRM) analysis of the relationship of verbal memory performance as indexed by the standardized CVLT-II Trials 1-5, diagnosis, and APOE- 44 status over time. We chose California Verbal Learning Test (CVLT) 1-5 as it represents the most reliable summary measure of immediate verbal memory. ${ }^{30}$ For fixed effects, we entered diagnosis (FEP vs CTRL) and $A P O E-\varepsilon 4$ status ( $A P O E-\varepsilon 4$ carriers vs non- $A P O E-\varepsilon 4$ carriers) as between-subjects and time (baseline vs follow-up) as within-subjects factor with the triple interaction term included in the model. Age was entered as a covariate. An unstructured covariance matrix was assumed. Visual inspection of residual plots did not reveal any obvious deviations from homoscedasticity or normality.

\section{Results}

\section{Verbal memory impairment in FEP}

A repeated measures ANOVA was conducted with withinsubjects factor (ie, standardized CVLT-II performance on six measures: Trial 1, Trial 5, short-delay free recall, shortdelay cued recall, long-delay free recall, long-delay cued recall) and one between-subjects factor (ie, FEP and CTRL). The test for parallelism indicated that the null hypothesis is tenable and both profiles are coincident $(F[5,118]=1.16$, $\left.P=0.30, \eta^{2}=0.05\right)$. The test for flatness indicated that flatness is not tenable $\left(F[5,118]=2.42, P=0.04, \eta^{2}=0.09\right)$. Follow-up comparisons for each pairwise difference were nonsignificant after Bonferroni multiple comparisons correction. A main effect of group was observer, with CVLT scores being significantly lower in $\operatorname{FEP}\left(F[1,122]=50.8, P<0.00001, \eta^{2}=0.29\right)$. Figure 1 displays the profile scores for both groups.

\section{Change in verbal memory performance over time}

MMRM analysis revealed a main effect of group on CVLT-II Trials $1-5(\mathrm{FEP}=43.30$ vs $\mathrm{CTRL}=58.25 ; F[1,119.7]=42.97$; $P<0.001$ ) but no main effect for $A P O E-\varepsilon 4$ ( $A P O E-\varepsilon 4$ carriers $=52.27$ vs $A P O E-\varepsilon 4$ noncarriers $=49.28 ; F[1,119.7]=1.72$; $P=0.19$ ) or time (baseline $=51.05$ vs follow-up $=50.49$; $F[1,109.2]=0.17 ; P<0.68)$. However, MMRM analyses revealed an interaction of $A P O E-\varepsilon 4$ by group by time $(F[4$, $116.2]=2.73, P=0.033)$. We followed up on this interaction by plotting the marginal means in memory performance across time by subgroup as shown in Figure 2. ${ }^{33}$ Indeed, visual inspection of this plot clearly reveals that the effect is mostly driven by change over time in FEP who are $A P O E-\varepsilon 4$

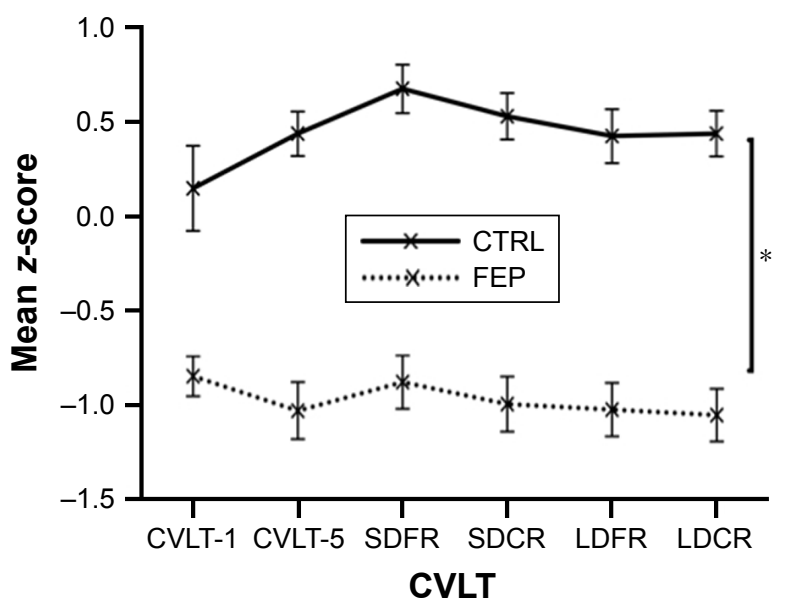

Figure I Performance profiles on the CVLT-II in FEP patients and healthy CTRL participants.

Notes: CVLT scores being significantly lower in FEP than in CTRL $(* P<0.001)$. Profiles were coincident for both groups. Profiles were not flat $(P=0.04)$, but follow-up comparisons for each pairwise difference were nonsignificant after Bonferroni multiple comparisons correction.

Abbreviations: CTRL, control; CVLT, California Verbal Learning Test; FEP, firstepisode psychosis; LDFR, long-delay free recall; LDCR, long-delay cued recall; SDFR, short-delay free recall; SDCR, short-delay cued recall.

carriers, showing that this group verbal memory improves at follow-up. Informed by this visual analytic technique, we followed up with post hoc analyses on FEP who were $A P O E-\varepsilon 4$ carriers using a paired $t$-test. Results showed a significant difference of verbal memory performance, improvement, at follow-up in FEP- $A P O E-\varepsilon 4$ carriers (baseline $=42.92$;

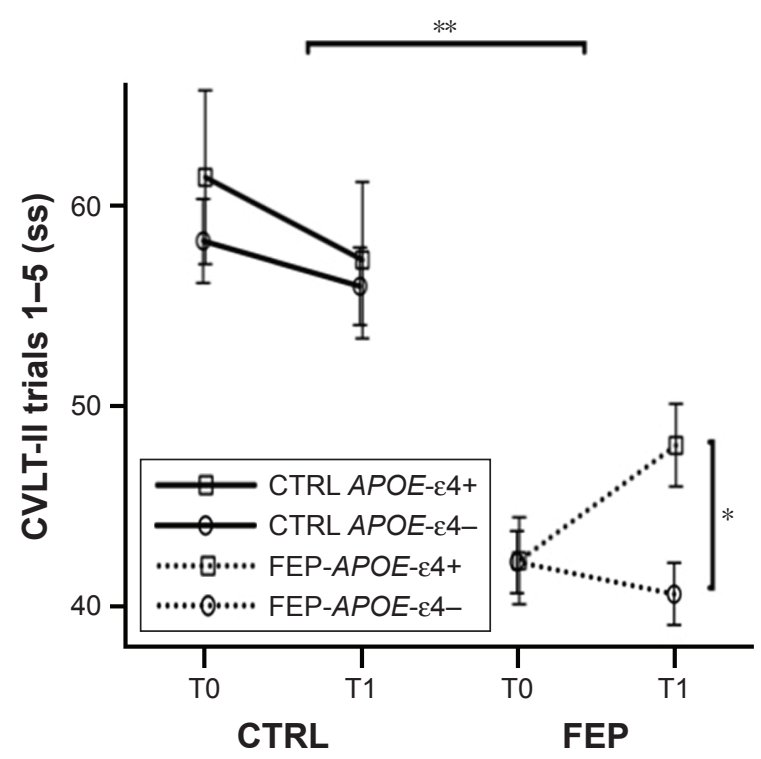

Figure 2 CVLT-II trials I-5 standardized scores in CTRLs and FEP patients at baseline (T0) and 9-12-month follow-up (TI).

Notes: FEP patients showed impaired verbal memory compared to CTRLs $(* * P<0.000 \mathrm{I})$, and there was an interaction of APOE- $\varepsilon 4$ by group by time $(P=0.033)$. Post hoc analyses on FEP who were APOE- $\varepsilon 4$ carriers showed a significant difference of verbal memory performance at follow-up in FEP-APOE- $\varepsilon 4$ carriers $(* P=0.025)$. Abbreviations: CTRL, control; CVLT, California Verbal Learning Test; FEP, firstepisode psychosis. 
follow-up $=48.36 ; t=-2.40, \mathrm{df}=24, P=0.025)$. There was no difference in lapse of time of cognitive testing between diagnostic groups, or there was a difference between $A P O E-\varepsilon 4$ carriers and noncarriers.

\section{Effect of medication on verbal memory performance and APOE- $\varepsilon 4$}

Medications at the time of cognitive testing were similar in FEP-APOE- $\varepsilon 4$ carriers vs FEP- $A P O E-\varepsilon 4$ noncarriers (Table 2). There was no significant correlation of total accumulated dose of antipsychotic at baseline in chlorpromazine (CPZ) equivalents and verbal memory performance at baseline (CPZ dose vs CVLT-II Trials $1-5, r=0.02, P=0.88$ ), or was mean CVTL-II Trials 1-5 score statistically different as a function of treatment (one-way ANOVA, olanzapine $=44.23$ vs risperidone $=43.11$ vs naïve $=41.38 ; F=0.323, \mathrm{df}=2,73$; $P=0.725)$. The percentage change of CPZ dose between baseline and follow-up was not statistically correlated with the percentage change in verbal memory performance between baseline and follow-up (percentage change CPZ dose vs percentage change CVLT-II Trials $1-5, r=-0.01, P=0.94$ ). $A P O E-\varepsilon 4$ status was not statistically correlated with the percentage of change in $\mathrm{CPZ}$ dose $(r=0.06, P=0.7)$. Also, prior work by our group showed no significant correlation of anticholinergic load and performance on CVLT-II Trials $1-5$ at follow-up. ${ }^{34}$

\section{Verbal memory performance and APOE- $\varepsilon 4$ effects on clinical outcomes}

There were no significant differences between FEP-APOE- $\varepsilon 4$ carriers and noncarriers in total or subscale Positive and Negative Syndrome Scale (PANSS) scores, clinical global impression scores, or level of functioning on the role functioning scale at baseline (Table 2). All measures aimed at ascertaining premorbid aspects such as mean age of first decline, mean age of first psychosis, and mean duration of untreated psychosis were similar in both groups (Table 2).

The percentage change in verbal memory performance between baseline and follow-up did not correlate with the percentage change in PANSS, Global Assessment of Functioning (GAF), clinical global impression (CGI), or Role Functioning Scale (RFS). APOE- $\varepsilon 4$ status did not correlate with percentage of change in PANSS, GAF, CGI, or RFS, but $A P O E-\varepsilon 4$ carrier status was associated with verbal memory performance percentage change $(r=0.32, P=0.008)$.

Better verbal memory performance at follow-up was correlated with less psychopathology (CVLT-II Trials $1-5$ vs PANSS total score $r=-0.24 ; P=0.047$ ) as well as less global severity (CVLT $1-5$ vs CGI $r=-0.3 ; P=0.013$ ). There was a strong nonsignificant trend showing better verbal memory at follow-up was correlated with better social functioning (CVLT-II Trials $1-5$ vs RFS score $r=0.23, P=0.058$ ). Post hoc correlation tests for the subscales of PANSS and RFS showed that better verbal memory performance at follow-up was specifically associated with lower psychopathology scores on the negative subscale of PANSS ( $r=-0.35 ; P=0.003)$ and better extended social network relationships subscale of RFS $(r=0.25 ; P=0.037)$.

\section{Discussion}

The main finding, and most remarkable, of our work is that our data do not support a detrimental effect of carrying the $A P O E-\varepsilon 4$ allele on verbal memory in FEP. Furthermore, our data support an interaction effect between carrying the $A P O E-\varepsilon 4$ allele, FEP, and improvement in verbal memory over time. This is counterintuitive as there is no evidence supporting a protective effect of $A P O E-\varepsilon 4$ in any neuropsychiatric disorder. In fact, carrying the $A P O E-\varepsilon 4$ allele would appear to be an unspecific risk factor that worsens brain health as it has been shown to be detrimental in a wide array of conditions from Alzheimer's disease ${ }^{10}$ to traumatic brain injury, ${ }^{35,36}$ Pick's disease, ${ }^{37}$ synucleopathies, ${ }^{38}$ or late life depression. ${ }^{39}$ The principal proposed mechanism by which $A P O E-\varepsilon 4$ results in deleterious effects to the brain in pathological states points to the decreased efficiency of $A P O E-\varepsilon 4$ to transport cholesterol and the impact that this has on cellular homeostasis and repair mechanisms in the nervous system. ${ }^{7}$

A plausible hypothesis that would explain our results in FEP is the presence of an antagonistic pleiotropic phenomenon for the $A P O E-\varepsilon 4$ allele. Antagonistic pleiotropy is a concept from evolutionary biology and asserts that some genes that affect fitness (ie, survival and reproduction) may operate differently across the lifespan. This allows the possibility that a gene that would confer an advantage early in life by increasing the probability of reproduction and/or survival would later in life be detrimental by accelerating the senescence process. ${ }^{22}$ The first condition for $A P O E-\varepsilon 4$ to have an antagonistic pleiotropic effect, increase in fitness early in life, is supported by research showing different $A P O E$ alleles having different fertility rates in humans. ${ }^{23,24}$ In addition, there are data pointing to cognitive advantages early in life to support the argument of increased fitness associated with APOE- $\varepsilon 4$. Wright et al ${ }^{25}$ found that infant $A P O E-\varepsilon 4$ carriers had an advantage in early stages of development measured with the Bayley Scale of infant development compared to 
those who were non- $A P O E-\varepsilon 4$ carriers, hinting perhaps at early life brain development advantage of having $A P O E-\varepsilon 4$ allele. Filippini et $\mathrm{al}^{26}$ reported that healthy young $A P O E-\varepsilon 4$ carriers outperformed noncarriers on tests of memory and other cognitive domains. Interestingly, functional neuroimaging studies in young healthy volunteers have shown that the underlying mechanism is mediated by $A P O E-\varepsilon 4$ carriers recruiting additional frontal regions to achieve this advantage. ${ }^{26,40}$ Furthermore, recent research in younger healthy volunteers using fMRI while performing cognitive tasks has shown that cognitive performance declines with age in $A P O E-\varepsilon 4$ carriers $^{41}$ and, in older healthy $A P O E-\varepsilon 4$ carriers, the compensatory mechanism fails and differences in neurocognitive performance start to no longer favor $A P O E-\varepsilon 4$ carriers. The possibility of an antagonistic pleiotropic effect of $A P O E-\varepsilon 4$ in FEP not only is intriguing but could also be a key concept to reconcile aspects of neurodevelopmental and progressive deterioration etiopathogenic models in schizophrenia, into an integrated model. Therefore, our findings show that improvement in cognition over time in FEP-APOE- $\varepsilon 4$ carriers would be converging with prior evidence in other populations and expand it to providing evidence for APOE- $\varepsilon 4$ to have an antagonistic pleiotropic effect as well in a pathological state in young adulthood (ie, first-episode psychosis).

The mechanism underlying the interaction effect observed in our data merits further attention. Our data do not support a practice effect to explain the improvement in verbal memory in FEP- $A P O E-\varepsilon 4$ carriers only. First, we used two alternate versions of the CVLT-II to minimize practice effects, ${ }^{31}$ and participants were randomly assigned to receive those versions in a counterbalanced way. Furthermore, healthy volunteers clearly show that there is no improvement on verbal memory scores over time, and the non-statistically significant decrease in their performance is within the variability that could occur using the two alternate forms of CVLT-II. ${ }^{31}$ We extensively explored associations between pharmacological interventions and performance on verbal memory, and we found no association between type of medication or accumulated dose with verbal memory performance. Interestingly, associations between changes in lipid profile associated with antipsychotic medications and clinical improvements have been previously observed. ${ }^{42,43}$ This raises the question as to whether antipsychotic treatment might impact cholesterol transport efficiency as a function $A P O E$ allele. Unfortunately, our study did not include any data on lipid profiles and, thus, this hypothesis remains to be tested in future studies. Nonetheless, our results would suggest a complex relationship between antipsychotic treatment and cognitive improvement and could offer a framework to interpret the literature about this matter and its controversies such as whether second generation antipsychotics are superior or not. ${ }^{44,45}$

We explored whether the $A P O E-\varepsilon 4$ status was associated with outcomes on psychopathology, social functioning, and global clinical impression. $A P O E-\varepsilon 4$ status was not associated with any outcome measures during the study or there was any difference in terms of premorbid illness measures such as duration of untreated psychosis, age of onset, and age at first decline. Given the hypothesis of antagonistic pleiotropy, $A P O E-\varepsilon 4$ in this young population appears not be associated with a detrimental effect, but our data do not replicate those finding less positive symptoms in $A P O E-\varepsilon 4$ carriers ${ }^{46,47}$ but are convergent to findings from other groups. ${ }^{48-50}$ In addition, our data provide further support to the association of cognitive performance and negative symptoms. ${ }^{51}$ Furthermore, the correlation between verbal memory performance at follow-up and functioning in the domain of social networking on the RFS in our study adds support and replicates existing literature. ${ }^{52}$ The current data confirm significant impairment in measures of verbal memory in FEP patients (Figure 1).

Our results should be considered in the context of some limitations. Our sample was composed only of young individuals, limiting our ability to test the hypothesis that $A P O E-\varepsilon 4$ carriers having variable cognitive performance at different age stages. This certainly merits further research, and it could be remedied in future studies by including a group of older adults afflicted with chronic psychosis. Our sample size is small in comparison to large consortiums investigating genetic effects in schizophrenia. The number of $A P O E-\varepsilon 4$ homozygotes did not allow us to test for a dose effect of the $A P O E-\varepsilon 4$ allele and other more nuanced hypotheses. The current data are a complementary contribution to that of large consortiums in that our results could be a catalyst for a hypothesis of antagonistic pleiotropy to be tested in large consortium samples rather than only using data-driven strategies.

\section{Acknowledgments}

Funding was provided by a grant from the Canadian Institutes of Health Research (NET-54013), the BC Mental Health and Addictions Services, and the Michael Smith Foundation for Health Research. WGH was supported by the Jack Bell Chair in Schizophrenia.

\section{Disclosure}

GWM received consulting or advisory board fees from AstraZeneca, Janssen, Eli Lilly, and Novartis, lecture fees 
from GlaxoSmithKline, as well as grant support from AstraZeneca, BMS, Janssen, and Pfizer. LCK received advisory board fees from Bristol Myers Squibb and Pfizer Canada as well as grant support from AstraZeneca. WGH received consulting fees or sat on paid advisory boards for the Canadian Agency for Drugs and Technology in Health, the Centre for Drug Research and Development, AlphaSights, In Silico, and Otsuka/Lundbeck and is supported by the Jack Bell Chair in Schizophrenia. The other authors report no other conflicts of interest in this work.

\section{References}

1. Seidman LJ. Schizophrenia and brain dysfunction: an integration of recent neurodiagnostic findings. Psychol Bull. 1983;94(2):195-238.

2. Green MF. What are the functional consequences of neurocognitive deficits in schizophrenia? Am J Psychiatry. 1996;153(3):321-330.

3. Mesholam-Gately RI, Giuliano AJ, Goff KP, Faraone SV, Seidman LJ. Neurocognition in first-episode schizophrenia: a meta-analytic review. Neuropsychology. 2009;23(3):315-336.

4. Heinrichs RW, Zakzanis KK. Neurocognitive deficit in schizophrenia: a quantitative review of the evidence. Neuropsychology. 1998;12(3): 426-445.

5. Singh PP, Singh M, Mastana SS. APOE distribution in world populations with new data from India and the UK. Ann Hum Biol. 2006;33(3): 279-308.

6. Mauch DH, Nagler K, Schumacher S, et al. CNS synaptogenesis promoted by glia-derived cholesterol. Science. 2001;294(5545): 1354-1357.

7. Boyles JK, Zoellner CD, Anderson LJ, et al. A role for apolipoprotein E, apolipoprotein A-I, and low density lipoprotein receptors in cholesterol transport during regeneration and remyelination of the rat sciatic nerve. J Clin Invest. 1989;83(3):1015-1031.

8. Nathan BP, Jiang Y, Wong GK, Shen F, Brewer GJ, Struble RG. Apolipoprotein $\mathrm{E} 4$ inhibits, and apolipoprotein $\mathrm{E} 3$ promotes neurite outgrowth in cultured adult mouse cortical neurons through the low-density lipoprotein receptor-related protein. Brain Res. 2002;928(1-2):96-105.

9. Ignatius MJ, ShooterEM, Pitas RE, Mahley RW. Lipoprotein uptake by neuronal growth cones in vitro. Science. 1987;236(4804):959-962.

10. Strittmatter WJ, Saunders AM, Goedert M, et al. Isoform-specific interactions of apolipoprotein E with microtubule-associated protein tau: implications for Alzheimer disease. Proc Natl Acad Sci U S A. 1994;91(23): 11183-11186.

11. Engelman CD, Koscik RL, Jonaitis EM, et al. Interaction between two cholesterol metabolism genes influences memory: findings from the Wisconsin registry for Alzheimer's prevention. J Alzheimers Dis. 2013; 36(4):749-757.

12. Bondi MW, Salmon DP, Monsch AU, et al. Episodic memory changes are associated with the APOE-epsilon 4 allele in nondemented older adults. Neurology. 1995;45(12):2203-2206.

13. Rapp MA, Schnaider-Beeri M, Purohit DP, et al. Cortical neuritic plaques and hippocampal neurofibrillary tangles are related to dementia severity in elderly schizophrenia patients. Schizophr Res. 2010;116(1): 90-96.

14. Kampman O, Anttila S, Illi A, et al. Apolipoprotein E polymorphism is associated with age of onset in schizophrenia. J Hum Genet. 2004;49(7): 355-359.

15. Harrington CR, Roth M, Xuereb JH, McKenna PJ, Wischik CM. Apolipoprotein $\mathrm{E}$ type epsilon 4 allele frequency is increased in patients with schizophrenia. Neurosci Lett. 1995;202(1-2):101-104.

16. Martorell L, Virgos C, Valero J, et al. Schizophrenic women with the APOE epsilon 4 allele have a worse prognosis than those without it. Mol Psychiatry. 2001;6(3):307-310.
17. Allen NC, Bagade S, McQueen MB, et al. Systematic meta-analyses and field synopsis of genetic association studies in schizophrenia: the SzGene database. Nat Genet. 2008;40(7):827-834.

18. Thibaut F, Coron B, Hannequin D, et al. No association of apolipoprotein epsilon 4 allele with schizophrenia even in cognitively impaired patients. Schizophr Res. 1998;30(2):149-153.

19. Sáiz PA, Morales B, G-Portilla MP, et al. Apolipoprotein E genotype and schizophrenia: further negative evidence. Acta Psychiatr Scand. 2002;105(1):71-75.

20. González-Castro TB, Tovilla-Zárate CA, Hernández-Díaz Y, et al. No association between ApoE and schizophrenia: evidence of systematic review and updated meta-analysis. Schizophr Res. 2015;169(1-3):355-368.

21. Hill WD, Davies G; CHARGE Cognitive Working Group, Liewald DC, McIntosh AM, Deary IJ. Age-dependent pleiotropy between general cognitive function and major psychiatric disorders. Biol Psychiatry. 2016;80(4):266-273.

22. Williams GC. Pleiotropy, natural selection, and the evolution of senescence. Evolution. 1957;11(4):398.

23. Corbo RM, Scacchi R, Cresta M. Differential reproductive efficiency associated with common apolipoprotein e alleles in postreproductiveaged subjects. Fertil Steril. 2004;81(1):104-107.

24. Jasienska G, Ellison PT, Galbarczyk A, et al. Apolipoprotein E (ApoE) polymorphism is related to differences in potential fertility in women: a case of antagonistic pleiotropy? Proc Biol Sci. 2015;282(1803): 20142395.

25. Wright RO, Hu H, Silverman EK, et al. Apolipoprotein E genotype predicts 24-month bayley scales infant development score. Pediatr Res. 2003;54(6):819-825.

26. Filippini N, MacIntosh BJ, Hough MG, et al. Distinct patterns of brain activity in young carriers of the APOE-epsilon4 allele. Proc Natl Acad Sci U S A. 2009;106(17):7209-7214.

27. Tuminello ER, Han SD. The apolipoprotein e antagonistic pleiotropy hypothesis: review and recommendations. Int J Alzheimers Dis. 2011; 2011:726197.

28. Uttl B. North American adult reading test: age norms, reliability, and validity. J Clin Exp Neuropsychol. 2002;24(8):1123-1137.

29. Kaufman AS, Kaufman NL. Kaufman brief intelligence test, Second Edition. John Wiley \& Sons, Inc. 2013.

30. Delis DC, Kramer JH, Kaplan E, Ober BA. California Verbal Learning Test - Second Edition. Adult Version. Manual. San Antonio, TX: Psychological Corporation; 2000.

31. Woods SP, Delis DC, Scott JC, Kramer JH, Holdnack JA. The California Verbal Learning Test - second edition: test-retest reliability, practice effects, and reliable change indices for the standard and alternate forms. Arch Clin Neuropsychol. 2006;21(5):413-420.

32. Heinrichs RW, Parlar M, Pinnock F. Normal-range verbal-declarative memory in schizophrenia. Neuropsychology. 2017;1(2):1-9.

33. Jaccard J, Turrisi R. Interaction Effects in Multiple Regression. 1st ed. Thousand Oaks, CA: SAGE; 2011.

34. Baitz HA, Thornton AE, Procyshyn RM, et al. Antipsychotic medications: linking receptor antagonism to neuropsychological functioning in first episode psychosis. J Int Neuropsychol Soc. 2012;18(4):717-727.

35. Crawford FC, Vanderploeg RD, Freeman MJ, et al. APOE genotype influences acquisition and recall following traumatic brain injury. Neurology. 2002;58(7):1115-1118.

36. Town T, Fallin D, Crawford F, Walsh S, Solomon R, Mullan M. Lack of association between the apolipoprotein E epsilon4 allele (APOE epsilon4) and chronic schizophrenia. Am J Med Genet. 1997;74(4):451-452.

37. Kálmán J, Juhász A, Majtényi K, et al. Apolipoprotein E polymorphism in Pick's disease and in Huntington's disease. Neurobiol Aging. 2000;21(4):555-558.

38. Tsuang D, Leverenz JB, Lopez OL, et al. APOE $\varepsilon 4$ increases risk for dementia in pure synucleinopathies. JAMA Neurol. 2013;70(2):223-228.

39. Zubenko GS, Henderson R, Stiffler JS, Stabler S, Rosen J, Kaplan BB. Association of the APOE epsilon 4 allele with clinical subtypes of late life depression. Biol Psychiatry. 1996;40(10):1008-1016. 
40. Filippini N, Ebmeier KP, MacIntosh BJ, et al. Differential effects of the APOE genotype on brain function across the lifespan. Neuroimage. 2011;54(1):602-610.

41. Nichols LM, Masdeu JC, Mattay VS, et al. Interactive effect of apolipoprotein e genotype and age on hippocampal activation during memory processing in healthy adults. Arch Gen Psychiatry. 2012;69(8): 804-813.

42. Procyshyn RM, Wasan KM, Thornton AE, et al; Clozapine and Risperidone Enhancement Study Group. Changes in serum lipids, independent of weight, are associated with changes in symptoms during long-term clozapine treatment. J Psychiatry Neurosci. 2007;32(5):331-338.

43. Procyshyn RM, Honer WG, Barr AM. Do serum lipids predict response to clozapine treatment? J Psychiatry Neurosci. 2009;34(2):168.

44. Harvey PD, Green MF, McGurk SR, Meltzer HY. Changes in cognitive functioning with risperidone and olanzapine treatment: a large-scale, double-blind, randomized study. Psychopharmacology (Berl). 2003; 169(3-4):404-411.

45. Keefe RSE, Bilder RM, Davis SM, et al; CATIE Investigators; Neurocognitive Working Group. Neurocognitive effects of antipsychotic medications in patients with chronic schizophrenia in the CATIE trial. Arch Gen Psychiatry. 2007;64(6):633-647.
46. Al-Asmary SM, Kadasah S, Arfin M, Tariq M, Al-Asmari A. Apolipoprotein E polymorphism is associated with susceptibility to schizophrenia among Saudis. Arch Med Sci. 2015;11(4):869-876.

47. Pickar D, Malhotra AK, Rooney W, Breier A, Goldman D. Apolipoprotein E epsilon 4 and clinical phenotype in schizophrenia. Lancet. 1997;350(9082):930-931.

48. Rietschel M, Krauss H, Müller DJ, Nöthen MM, Macciardi F. Apolipoprotein E epsilon 4 and clinical phenotype in schizophrenia. Lancet. 1997;350(9094):1857-1858.

49. Ohara K, Nagai M, Ohara K. Apolipoprotein E epsilon 4 and clinical phenotype in schizophrenia. Lancet. 1997;350(9094):1857.

50. Igata-Yi R, Igata T, Ishizuka K, et al. Apolipoprotein E genotype and psychosis. Biol Psychiatry. 1997;41(8):906-908.

51. Harvey PD, Koren D, Reichenberg A, Bowie CR. Negative symptoms and cognitive deficits: what is the nature of their relationship? Schizophr Bull. 2006;32(2):250-258.

52. Addington J, McCleary L, Munroe-Blum H. Relationship between cognitive and social dysfunction in schizophrenia. Schizophr Res. 1998;34(1-2):59-66. 


\section{Supplementary materials Detailed material and methods}

Inclusion criteria for patients for this study were presentation of Diagnostic and Statistical Manual IV (DSM-IV) defined psychotic disorders and fluency in English. Exclusion criteria were as follows: 1) history of significant head injury defined by loss of consciousness $>5$ minutes, 2 ) history of neurodevelopmental disorder or central nervous system infection, 3 ) history of inhalant intoxication, 4) inability to provide full written consent, and 5) intelligence quotient $<75$. Inclusion and exclusion criteria were similar for healthy volunteers, except history of major psychiatric disorder.

DSM-IV diagnoses were based on the Structured Clinical Interview for DSM-IV, a review of clinical case notes, and interviews with one or more family members. This information was presented at a case conference, and a consensus diagnosis made by at least two psychiatrists and a clinical psychologist. ${ }^{1}$ A detailed review of past and current substance use was obtained at baseline and follow-up. The Positive and Negative Syndrome Scale (PANSS) ${ }^{2}$ estimated psychiatric symptom severity at baseline and follow-up. A five-factor solution was computed at baseline and follow-up based on a meta-analysis of first episode psychosis. ${ }^{3}$ Chlorpromazine equivalents were computed according to Woods. ${ }^{4}$ Level of functioning was indexed at baseline using the Social and Occupational Functioning Assessment Scale ${ }^{1}$ and the Role Functioning Scale (RFS). ${ }^{5}$

\section{Statistical analyses}

Chi-square tests were used to test the null hypothesis of equality of proportions in different groups (two levels of sex, men and women; four levels of ethnicity, Caucasian,
Asian, and others; four levels of diagnoses, schizophrenia, schizoaffective, affective psychosis, and others; two levels of APOE- $\varepsilon 4$ status, APOE- $\varepsilon 4$ carriers and APOE- $\varepsilon 4$ noncarriers; and four levels of type of medication, naïve, olanzapine, risperidone, and others). Independent $t$-tests were used to test the null hypothesis of equal means in different independent groups for age, years of education, NAART, K-Bit, PANSS, clinical global impression (CGI), RFS, age at first decline, age at first psychotic symptoms, and duration of untreated psychosis.

Correlation analyses were used to explore the relationship between APOE- $\varepsilon 4$ status, accumulated chlorpromazine dose, California Verbal Learning Test (CVLT) 1-5, PANSS, CGI, RFS at follow up as well as ratios of change for CVLT 1-5, PANSS, CGI, and RFS. Post hoc correlation analyses were used to explore the relationship between CVLT 1-5 at follow-up and the subscales of both the PANSS and the RFS. Correlation analyses were not corrected for multiple comparison. ${ }^{6}$

\section{References}

1. American Psychiatric Association. Diagnostic and Statistical Manual of Mental Disorders, 4th Edition, Text Revision (DSM-IV-TR). American Psychiatric Association; 2000.

2. Kay SR, Fiszbein A, Opler LA. The positive and negative syndrome scale (PANSS) for schizophrenia. Schizophr Bull. 1987;13(2):261-276.

3. Langeveld J, Andreassen OA, Auestad B, et al. Is there an optimal factor structure of the Positive and Negative Syndrome Scale in patients with first-episode psychosis? Scand J Psychol. 2013;54(2):160-165.

4. Woods SW. Chlorpromazine equivalent doses for the newer atypical antipsychotics. J Clin Psychiatry. 2003;64(6):663-667.

5. Goodman SH, Sewell DR, Cooley EL, Leavitt N. Assessing levels of adaptive functioning: the Role Functioning Scale. Community Ment Health J. 1993;29(2):119-131.

6. Rothman KJ. No Adjustments Are Needed for Multiple Comparisons. Epidemiology. 1990;1(1):43-46.
Neuropsychiatric Disease and Treatment

\section{Publish your work in this journal}

Neuropsychiatric Disease and Treatment is an international, peerreviewed journal of clinical therapeutics and pharmacology focusing on concise rapid reporting of clinical or pre-clinical studies on a range of neuropsychiatric and neurological disorders. This journal is indexed on PubMed Central, the 'PsycINFO' database and CAS,

\section{Dovepress}

and is the official journal of The International Neuropsychiatric Association (INA). The manuscript management system is completely online and includes a very quick and fair peer-review system, which is all easy to use. Visit http://www.dovepress.com/testimonials.php to read real quotes from published authors. 